\title{
SPEECH QuALITY EVALUATION BASED CODEC FOR VOIP OVER 802.11P
}

\author{
Said El Brak ${ }^{1}$, Mohammed Bouhorma ${ }^{2}$, Mohamed El Brak ${ }^{3}$ and Anouar Bohdhir ${ }^{4}$ \\ ${ }^{1,2,4}$ LIST Laboratory, Abdelmalek Essaadi University, FSTT, Tangier, Morocco \\ elbraks@gmail.com \\ ${ }^{3}$ ITS Laboratory, Abdelmalek Essaadi University, Faculty of Science, Tetouan, Morocco \\ melbrak@yahoo.fr
}

\begin{abstract}
Voice over Internet Protocol (VoIP) may provide good services through Vehicular ad hoc networks (VANETs) platform by providing services to many application scenarios range from safety to comfort. However, VANETs networks introduce many challenges for supporting voice with QoS requirements. In this paper, our study is based on Inter-Vehicle voice streaming rely on multi-hop fashion. For this task, a performance evaluation of various audio CODECs will be analyzed by mean of simulations. Furthermore, we test the impact of network environment on QoS metrics. To achieve good results, CODECs behaviour is tested by using mobility information obtained from vehicular traffic generator. The mobility model is based on the real road maps of an urban environment. Focusing on inter-vehicular voice traffic quality, we provide simulations results in terms of both user level (MOS) metrics and network level ( such as Losses). According to this performance evaluation, we show that G.723.1 CODEC worked well in the urban VANET environment.
\end{abstract}

\section{KEYWORDS}

Vehicular ad hoc networks (VANETs), Voice over IP (VoIP), Quality of Service (QoS), CODECs, Vehicular Traffic Generator, $n s-2$

\section{INTRODUCTION}

Mobile ad hoc networks (MANETs) are autonomous networks consisting of mobile nodes equipped with wireless communication and networking capabilities, communicating without any network centralized infrastructure [1]. The existence of these networks opens the way for a large range of application scenarios such as: Home and enterprise networking, emergency services, Entertainment Sensor networks and Vehicular services, etc.

VANETs are specific class of MANETs providing real-time information that could be useful for keeping people connected in urban environments or highways in a clear advance to safer and comfort driving. However, such networks introduce several constraints like the high mobility of the nodes, frequently changing topology, hard delay, etc [2]. These characteristics distinguish them from other mobile ad hoc networks.

Voice over Internet Protocol (VoIP) is one of the most important technologies that allow making voice calls through Internet connection. As it is well known, the quality of service is important for VoIP applications. They especially require limited end-to-end delay and a low packet loss rate [3]. To ensure quality voice communication, a suitable voice coding techniques (CODECs) are needed [4]. The primary function of CODECs is to perform analog/digital voice signal conversion and digital compression. 
Simulation has become an indispensable tool which allows building a dedicated VANET for the network performance evaluation. In this study, Network Simulator 2 (ns-2) [5] is used to run several simulations. Additionally, ns2voip++ [6] modules was employed to generate voice traffic with the proactive routing protocol OLSR. The VANET instances have been defined by using real data (roads specification and mobility models) concerning an urban area of Tangier city, in Morocco. In turn, the network definition includes the specification of real IEEE $802.11 \mathrm{p}$ [7] devices and a trustworthy wave urban Nakagami propagation model [8]. In order to provide an accurate quantitative evaluation of different voice CODECs, the test is performed by a number of realistic VANET simulations under different conditions. The main contributions of this work are:

- Generating several urban VANET instances following real data,

- Including IEEE $802.11 \mathrm{p}$ connectivity to achieve accurate simulations,

- Studying different QoS metrics to evaluate CODECs performances,

- Testing how VANET environment can influence the human perceived quality of VoIP call,

This paper is organized as follows: Section 2 overviews VANETs and provides their technical aspects such as architecture, routing and MAC protocols. Section 3 presents VoIP Service over vehicular networks. Section 4 shows the methodology for the simulation. Results and performance analysis, and comparisons are presented in section 5. Finally, conclusions and future work are drawn in Section 6.

\section{BACKGROUND OF VEHICULAR AD HOC NETWORKS}

Vehicular Ad hoc NETworks (VANETs) aim to make road more secure and time on the road more enjoyable. From the vehicular communication perspective, VANETs system architectures (Figure. 1) can be categorized into vehicle to infrastructure communication (V2I) systems, and inter-vehicle communication (V2V) systems [9].

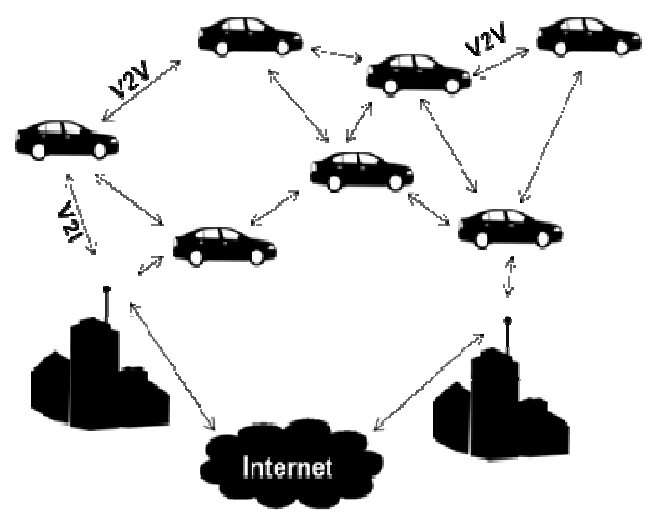

Figure 1. VANETs system architecture

Application perspective: VANETs provide variety of applications which can be categorized into two groups as safety and comfort applications. The first one focuses on relieving the vehicletraffic congestion and improving operations management in support of public safety goals, such as collision avoidance. This application is delay sensitive and should be given priority over non- 
safety applications. Second group focuses on vehicle user's entertainment like: multi-user games, wireless P2P networking, Internet access and touristic information [10]. In this context, voice traffic provides services to many VANETs application, especially in emergency intervehicle communications like: Police cars, Ambulances and fire apparatus etc. Note that in most emergency cases very remote areas and disaster struck lack telecommunication systems.

Network perspective: The network layer provides wireless multi-hop communications. VANET considered as a special implementation of MANET, This suggests the applicability of most MANET routing protocols in VANET, except high nature of unpredictability and the high speed mobility of VANETs. Otherwise, MANET routing protocols should be redesigned in order to behave with the unique VANETs characteristics and needs. Recent studies show that proactive routing protocols as OLSR generally outperform the reactive ones in terms of network goodput and end-to-end delay (that is an important feature for VANET application) [11]. OLSR protocol exhibits a series of features that make it well-suited for VoVANs. Hence, the protocol has been chosen for this work.

MAC and PHY protocol issues: In order to efficiently share the medium in VANETs networks, various MAC protocols have been proposed. Currently, DSRC (Dedicated Short-Range Communication) specifically for VANETs. $802.11 \mathrm{p}$ is an IEEE standard which enhances wireless access functionality that will permit applications for rapidly changing vehicular network environments. The Enhanced Distributed Channel Access (EDCA) has been used in the Medium Access Control protocol in IEEE 802.11p, and uses mechanism originally provided by IEEE 802.11e [12] where different Arbitration Inter Frame Space (AIFS) and Contention Window $(\mathrm{CW})$ values are chosen for different application categories (ACs). In the physical layer, IEEE-802.11p operates at 5.9GHz band (U.S) and 5.8GHz band (Japan and Europe) with $75 \mathrm{MHz}$ bandwidth divided in seven channels each with $10 \mathrm{MHz}$ frequency band. Control channel are in the middle and other are the service channels.

Mobility issues: Mobility models for MANETs may not be directly applicable to VANETs. Vehicular environment presents different requirements, such as multi-path fading and roadside obstacles, constrained road topology, varying vehicular mobility and speed, traffic congestion and lights, etc [13]. However, we need to generate a mobility model that is as realistic as an actual vehicular environment to achieve good results from VANET simulations, Currently, different road traffic generators have been used in order to generate the realistic simulation mobility models where vehicles move following the real traffic rules previously presented. [14] Provides a comparative study of various publicly available VANET simulators and mobility generators that are currently in use by the research community.

\section{VOIP OVER VANETS (VOVAN)}

As illustrated on Figure 2, the speech source alternates between talking and silence period which is typically considered to be exponentially distributed. The speech will enter to the digitalization process that is composed of sampling, quantization and encoding. The encoded speech is then packetized into packets of equal size preparing them for transmission over IP network. In the receiver side, encoded speech will be comprised by the payload for certain duration depends on the codec deployed, than reverse process is performed (depacketized and decoded). 


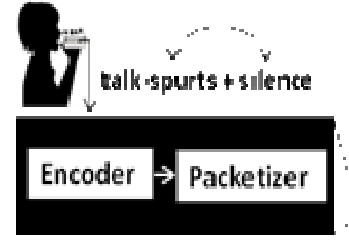

Sender

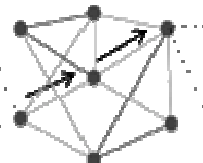

Playout

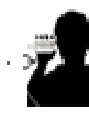

Decoder

Figure 2. VoIP system over MANETs [3]

The first step for voice communication is the application of a voice CODEC (COder/DECoder) which is a device and/or software program that is used typically to digitally encode an analog voice waveform. Various encoding techniques have been developed and standardized by the International Telecommunications Union (ITU-T). Table 1 shows some of the commonly used ITU-T standard CODECs, and lists their attributes. Generally, Coding process involves converting the incoming analog voice pattern into a digital stream and converting that digital stream back to an analog voice pattern at the ultimate destination. The objective of a codec is to obtain the lowest rate bit stream possible after conversion without degrading the quality of the signal such that the received audio signal can be generated without noticeable differences in quality. CODECs generate constant bit-rate audio frames consisting of 40 bytes IP/UDP/RTP headers followed by a relatively payload. Voice traffic has a very stringent delay and packet loss constraint. However, CODECs add additional delay to the total network delay that will influence the speech quality.

Table 1. Voice CODECs description

\begin{tabular}{|c|c|c|c|c|}
\hline Codec & $\begin{array}{c}\text { Bit rate } \\
\text { (kbps) }\end{array}$ & $\begin{array}{c}\text { Sample size } \\
\text { (bytes) }\end{array}$ & $\begin{array}{c}\text { Packets } \\
\text { per second }\end{array}$ & $\begin{array}{c}\text { Payload size } \\
\text { (bytes) }\end{array}$ \\
\hline G.711 & 64 & 80 & 50 & 160 \\
\hline G.723.1 & 5.3 & 20 & 33.3 & 20 \\
\hline G.726 & 32 & 20 & 50 & 80 \\
\hline G.729A & 8 & 10 & 50 & 20 \\
\hline
\end{tabular}

Certainly, VANETs applications are concerned by transmitting voice between network entities. Vehicular Tele-emergency system is an application that need voice communication which is in most application cases, very remote areas and disaster struck areas lack telecommunication infrastructure (dead zones). Therefore, the investigation of vehicular ad hoc network for voice communication is needed. However, Multi-hop voice delivery through VANET is challenging since it must provide QoS provisioning by efficiently handling rapid topology changes and a fragmented network.

VoIP over MANETs (VoMAN) have been studied in [15] and shows how hops number affect significantly the QoS. VoIP applications impose diverse. Primary challenge in designing VoVAN is to provide good delay performance under the constraints of vehicular speeds, unreliable connectivity, and fast topological changes. Knowing that voice quality is mainly influenced by the choice of CODECs, a set of simulations was performed in order to test VoVAN performance with different voice encoder. The next section describes our measurement methodology. 


\section{Measurement Methodology}

VANETS performance evaluation is being studied by several researchers [17]. However, measurement of an actual VANET is expensive and infeasible. Therefore, simulation seems to be the most feasible solution. For this purpose, the discrete event network simulator ns-2 was used combined with road traffic generator.

\subsection{Vehicular mobility environment:}

Traffic simulator is needed to generate realistic vehicular mobility traces, used as an input for the network simulator. In our simulations, we use a microscopic vehicular traffic generator based on the car-following and lane-changing models proposed by Gipps [18]. This generator is used in conjunction with ns-2 and digital road maps from the TIGER (Topologically Integrated Geographic Encoding and Referencing) database.

To generate trace files reflecting vehicles movements considering a typical urban scenarios (portion of the area of Tangier city), presented in figure 3. The considered scenarios focus on the unicast transmission of voice signals between vehicles moving at rate of $0-50 \mathrm{~km} / \mathrm{h}$ during 200s, with an average inter-vehicle distance of 5 to 20 meters in increments of 7 meters. In order to analyze how various conditions affect the quality of the voice, two scenarios are considered. VoVAN is simulated with varying conditions which are network area sizes and traffic densities. VANET scenarios details are summarized in Table 2.

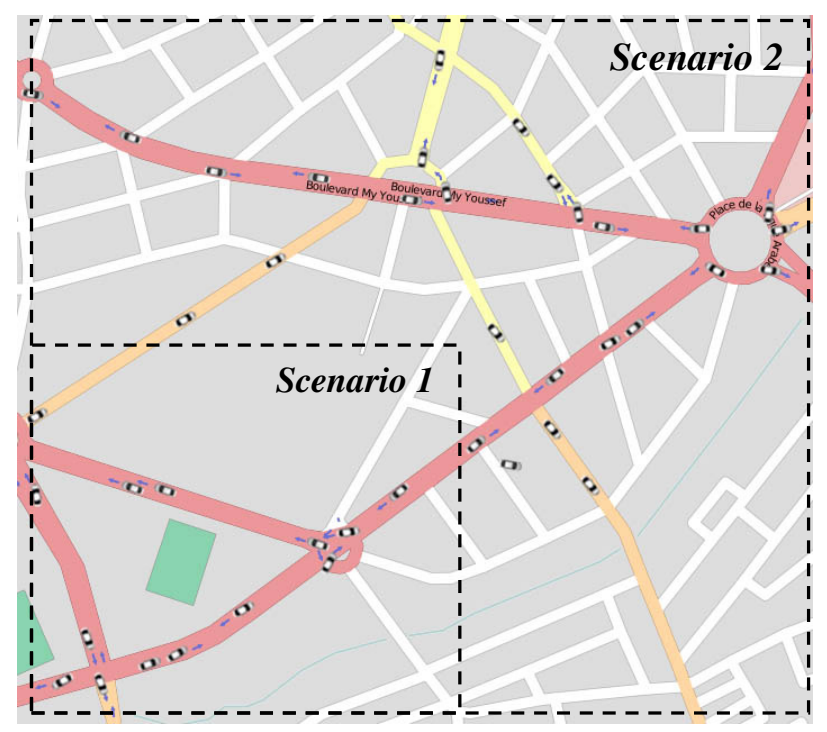

Figure 3. Tangier urban areas taken into account in our experiments

Table 2. VANET simulation scenarios

\begin{tabular}{|l|l|l|l|l|l|l|}
\cline { 2 - 7 } \multicolumn{1}{c|}{} & \multicolumn{3}{l|}{ Scenario 1} & \multicolumn{4}{c|}{ Scenario 2 } \\
\hline Area size & \multicolumn{2}{|c|}{$240 \mathrm{~m}^{2}$} & \multicolumn{4}{c|}{$800 \mathrm{~m}^{2}$} \\
\hline Vehicles & 10 & 20 & 30 & 30 & 40 & 60 \\
\hline $\begin{array}{l}\text { VoIP } \\
\text { Sources }\end{array}$ & 5 & 10 & 20 & 15 & 20 & 30 \\
\hline
\end{tabular}


International Journal of Wireless \& Mobile Networks (IJWMN) Vol. 5, No. 2, April 2013

\subsection{Network simulation Environment:}

Reflecting the network interactions in a trustworthy manner is an important issue in simulation. Ns-2 is a general networks simulator developed by Berkley University and has been widely used for MANETs. However, VANET simulation is fundamentally different from MANETs simulation, because in VANETs new MAC/PHY protocol is used. Hence, we use IEEE 802.11p standard which has been developed in recent ns-2 versions (2.34). Thus, the configuration is completed by introducing two new native modules: Mac802_11Ext and WirelessPhyExt that have been developed in [19].

Propagation signal modeling is a fundamental issue on wireless simulation studies. Recent research has shown that a fading radio propagation model, such as the Nakagami model, is best for simulation of a VANET environment [8]. As a result, the simulator has been configured to use this propagation model.

The network layer employs OLSR [20] routing protocol to compute the routing paths among the VANET nodes. OLSR parameters have been tuned by following specifications of [11]. Finally, the network workload is generated by a voice traffic generator (ns2voip++ module [6] with duration of 60s. The number of viop sources is dependent of urban VANET scenario and are performed during different moments of the simulation time. Each VoIP source is configured to draw the duration of the talk-spurt and silence periods from Weibull distribution. Additionally, scenarios have been experimented varying the voice CODECs: G.711, G.723.1, G.729A and G.726. Table 3 summarizes some important features of the network used in our VoVAN simulations.

Table 3. Simulation parameters

\begin{tabular}{|l|l|}
\hline Parameter & Value or Protocol \\
\hline Propagation model & Nakagami \\
\hline PHY/MAC layer & $802.11 p$ \\
\hline Network layer & Tuned OLSR \\
\hline Transport layer & RTP/UDP \\
\hline Application Layer & $n s 2$ viop ++ \\
\hline Voice CODECs & G.711, G.723.1, G.729A, G.726 \\
\hline VoIP Duration & $60 s$ \\
\hline Simulation time & $200 s$ \\
\hline
\end{tabular}

\subsection{Evaluation metrics:}

With the purpose of evaluating the performance of VoVAN system, three QoS metrics have been analyzed. These metrics are associated with user and network level that have a significant influence on perceived speech quality.

Delay: The network delay is defined as the interval between the frame arrival time at the application layer of a transmitter and the time at which the transmitter realizes that the transmitted frame has been successfully received by the receiver. This includes: packetization delay, queuing delay, transmission and propagation delay, and Play-out delay. According to ITU Recommendation [21], to achieve good transmission quality, a network delay of no more than $150 \mathrm{~ms}$ is required. If the delay exceeds $300 \mathrm{~ms}$, the quality of the VoIP stream is significantly degraded. 
Packet loss: Is the acceptable percentage of total packets sent, which are not received by the transport or higher layer agent at the packet's final destination node; while traversing a network, packets of data may pass through multiple processing stages as they are routed from source to destination. A loss of $5 \%$ or more is usually noticeable. Though VoIP applications tolerate packet loss up to $10 \%$, a packet loss of $1 \%$ still affects the quality of the VoIP stream [21].

Mean Opinion Score (MOS): used to express the human opinion about QoS. ITU-T P800 defines MOS as a subjective metric which estimates the user satisfaction by means of a score which varies from 1.0 (poor) to 5.0(best) [21]. MOS score is found by converting R-Factor scale obtained by the following expression:

$$
R=R O-I s-I d-I e+A
$$

Where:

- $\quad R$ represents the result voice quality (from 0 to 100 ),

- $R 0$ refers to noise ratio,

- Is characterizes the simultaneous impairment factor such as too load speech level,

- Id represents mouth-to-air delay,

- $\quad I e$ is the equipment impairment factor, and

- $A$ is the advantage of access.

\section{RESULTS AND DISCUSSION}

The overall performance of the four CODECs was tested using the average results obtained from 24 VANET scenarios defined in the specification presented above. The analysis focus on the results considering two urban scenario sizes (scenario 1 and 2). This section shows and discusses our simulation results investigating the impacts of the introduced QoS measured in terms of end-to-end delay, packet loss and MOS.

Average E2E delay metric is presented in Figure 4. G.723.1 and G.729A present the best performance with respect to other CODECs. These results are due to packet size. The larger packet size, the more time is required to process them. The relatively low packet size (20 bytes for G.723.1 and G.729A) and transfer rate make G.723.1 and G.729A the ideal CODECs. Otherwise, G.711 suffered higher delay than other coders for the reason that it has the larger packet size (160 bytes). In turn, the E2E delay is increased with size. 


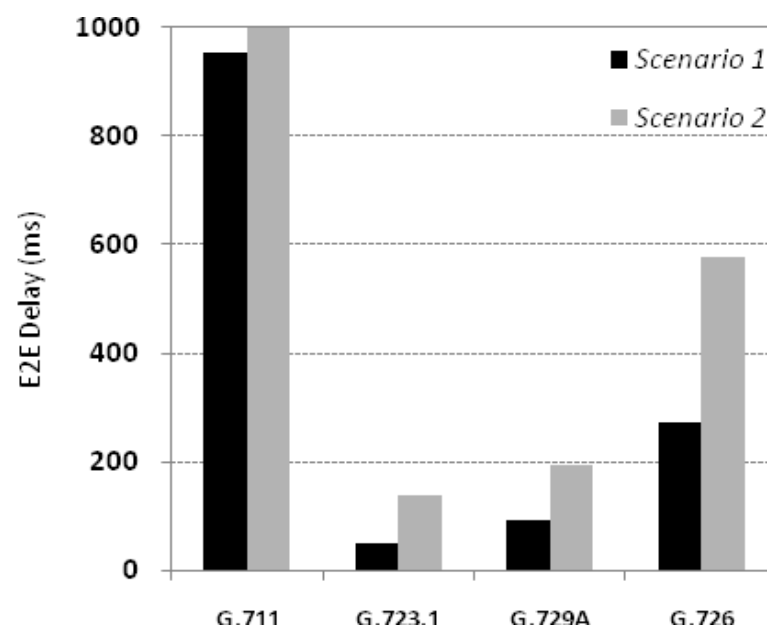

Figure 4. End-to-end delay for different audio CODECs

Examining the packet loss indicator for all CODECs (Figure 5), we can check that it exceed $10 \%$ which is the threshold for achievable voice communication. Firstly, these losses are due to the huge traffic introduced by the CODECs in one second, while each node is only able to handle 50 packets in its queue. Secondly, bases on ns-2 simulation trace results; losses are due to route discovery process (NRTE). While node spends some time to locate a route to destination, the VoIP source continues to produce packets. When route is not yet available, and the queue is full, packets on this one will be discarded. G.723.1 is moderately decreasing packet loss in the small scenario size because the traffic is slower (34 packet/sec) compared to other CODECs. Generally, losses increase in large scenario area because the number of connections increases.

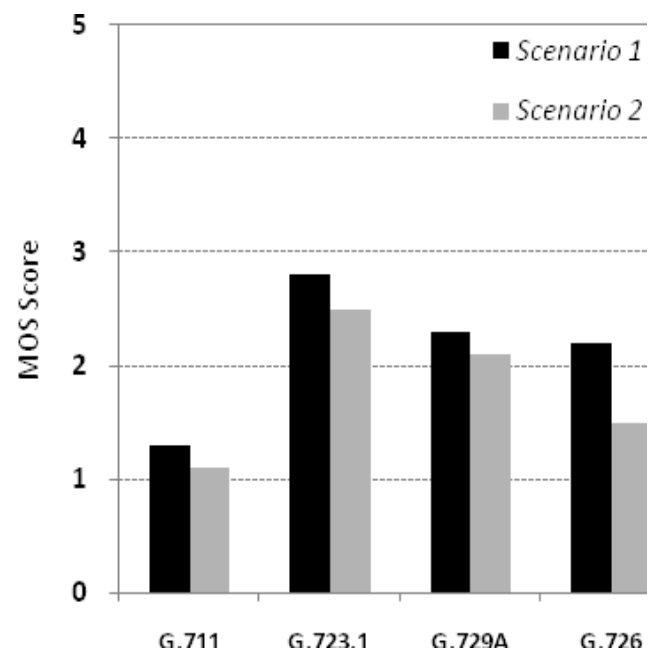

Figure 5. Packet loss for different audio codecs

The MOS is one of the most widely used QoS metric in VoIP applications, which help to computes a predictive estimation of the subjective voice quality. However, MOS is fundamentally affected by packet loss and delay. In figure 6, MOS is plotted for different CODECs. The best MOS value is 2.8 for G.723.1 which seems quietly acceptable. In general, 
MOS values are not satisfied with the call quality, mostly due to high loss rate. Another reason to that behavior is the fact that in urban VANET environment, the probability of link features tends higher because of fading radio propagation model. Finally, after studying the results, we have concluded that QoS decreased with the scenario size and VoVAN connections.

Very few papers exist about evaluating VoIP performance over VANETs. In [22] an experimental study of VoIP performance over V2V IEEE 802.11p links is presented; the authors conclude that performance is unsatisfactory because of packet loss; similar results are obtained with G.729A CODEC. In [23] the simulations for VoIP and Video traffic simulation over mobile tele-emergency system were investigated; the authors conclude that G.723.1 worked well in both small and medium scale network which nearly confirms our results.

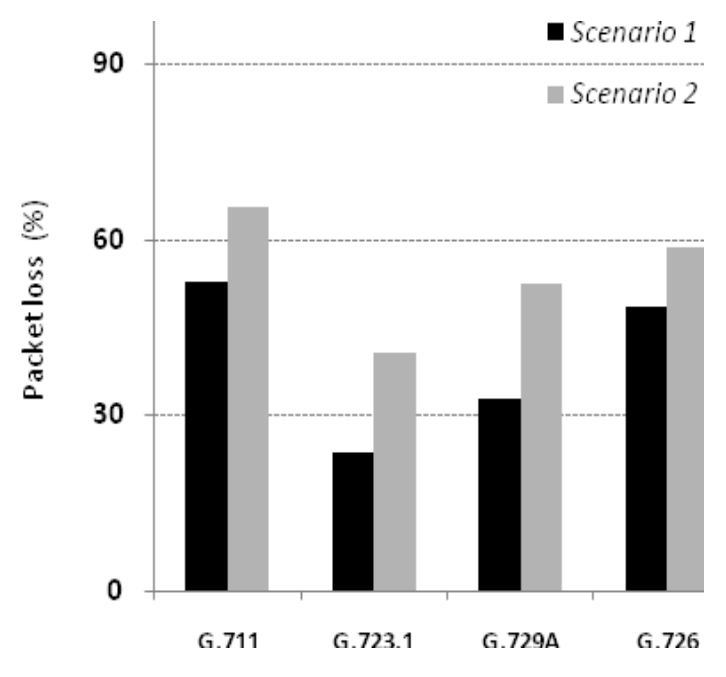

Figure 6

Figure 6. MOS score for different audio codecs

\section{CONCLUSION \& FUTURE WORKS}

VANET is an emerging field in networking area. Real-time voice transmission over such network is very much demanding and necessary, especially in VANET emergency scenarios. This work analyzed the performance evaluation of VoIP services in VANET in context of different voice CODECs. Considering inter-vehicle voice communication, impacts on quality of service metrics were addressed through simulations by means of ns- 2 over the OLSR Protocol with joint use of the Nakagami signal propagation model. A series of simulations have been carried out, based on real data of downtown of Tangier, (Morocco). The performance in terms of QoS has been measured using three metrics (E2E delay, packet loss and MOS). The empirical results presented in this paper showed performance of different audio CODECs, which may provide a strong reference to deployment of VoIP services through VANET, our conclusion are those:

- Generally, QoS decreased with the scenario size and VoVAN connections.

- G.723.1 presents the best optimal performance in terms of delay while G.711 is inappropriate to VANET.

- In term of packet losses, all CODECs exceed the acceptable threshold. 
- With regard to MOS metric, our study shows that G.723.1 provides an acceptable quality compared to other CODECs.

Finally, according to the results obtained in our work, in VANET environment CODECs must be more able to deal with delay and handle packet loss, since VANETs are high dynamic networks and urban environment affect the QoS.

As a matter of future works, we intend to design a QoS management system for VoVAN based on policies and CODECs adaptation, in order to adapt voice CODECs with VANET network environment. In addition, we are extending our experiments in highways, and planning outdoor test to validate the simulation results.

\section{REFERENCES}

[1] H. Ramin, "Ad-hoc Networks: Fundamental Properties and Network Topologies", Springer, Springer (2006) , p. I-XIII, 1-146. 2.

[2] S. Zeadally, R. Hunt, Y. Chen, A. Irwin, and A.Hassan, "Vehicular Ad Hoc Networks (VANETs): Status, Results, and Challenges," Telecommunication System, Vol. 51, Issue 2\&3, (Springer 2010).

[3] D. Minolli, E. Minoli, "Delivering Voice over IP Networks". Second Edition, Wiley Publishing, Inc. 2002.

[4] H. Dong, I.D. Chakares, C.-H. Lin, A. Gersho, E. Belding-Royer, U. Madhow, J.D. Gibson, Speech coding for mobile ad hoc networks, in: Proceedings of the Asilomar Conference on Signals, Systems, and Computers (ACSSC'03), vol. 1, 2003, pp. 280-284.

[5] The Network Simulator Project - ns-2. [online] Available in URL http://www.isi.edu/nsnam/ns/

[6] A. Bacioccola, C.Cicconetti, and G. Stea, "User-level performance evaluation of voip using ns-2". In the 2nd international conference on Performance evaluation methodolo, gies and tools. Nantes, France. 2007.

[7] D. Jiang, and L. Delgrossi, "IEEE 802.11p: Towards an international standard for wireless access in vehicular environments", Vehicular Technology Conference, pp. 2036 -2040. VTC Spring. IEEE. 2008.

[8] V. Taliwal, D. Jiang, H. Mangold, C. Chen, and R. Sengupta, "Empirical determination of channel characteristics for DSRC vehicle-to-vehicle communication". In Proceedings of the 1st ACM international workshop on Vehicular ad hoc networks, VANET '04, pages 88-88, NY, USA. 2004.

[9] U. Lee, R. Cheung and M. Gerla,. "Emerging Vehicular Applications. Vehicular Networks: From Theory to Practice”, Olariu, S., M. Aylene and C. Weigle (Eds.). CRC Press, USA., pp: 6-27. 2009

[10] G. M. T. Abdalla, M. A. Abu-Rgheff, and S. M. Senouci,. "Current Trends in Vehicular Ad Hoc Networks”, UbiCC Journal - Special issue of UbiRoads, ISSN Online 1992-8424, ISSN Print 19944608, 2007

[11]J. Toutouh, J. Garcia-Nieto, and E. Alba, "Optimal configuration of OLSR routing protocol for VANETs by means of Differential Evolution”. In 3rd International Conference on Metaheuristics and Nature Inspired Computing, META 2010, D’Jerba (Tunissia).

[12] T. B. Reddy, I. B.S. Karthigeyan, C. Manoj, S. R. Ram Murthy, "Quality of Service Provisioning in Ad hoc Wireless Networks: a Survey of Issues and Solutions” Ad Hoc Networks, 4, 83-124, 2006.

[13] C-K. Toh, “Ad Hoc Mobile Wireless Networks: Protocols and Systems”. Prentice Hall: Upper Saddle River, NJ, USA, 2001.

[14]F. J. Martinez, C. K. Toh, J. -C. Cano, C. T. Calafate, and P. Manzoni,. “A survey and comparative study of simulators for vehicular ad hoc networks (VANETs)," Wireless Communications and Mobile Computing, 2009. 
International Journal of Wireless \& Mobile Networks (IJWMN) Vol. 5, No. 2, April 2013

[15] S. El brak, M. Bouhorma, A.A. Boudhir,. "VoIP over MANET (VoMAN): QoS \& Performance Analysis of Routing Protocols for Different Audio Codecs", IJCA Journal , Volume 36, doi> 10.5120/4552-6449, 2011.

[16] J. Blum, A. Eskandarian, and L. Hoffman,. "Challenges of intervehicle ad hoc networks," IEEE Transactions on Intelligent Transportation Systems., vol. 5, no. 4, pp. 347-35, 2004.

[17]T. Murray, M. Cojocari, and H. Fu, "Measuring the performance of IEEE 802.11p using NS-2 simulator for vehicular networks," in IEEE International Conference on Electro/Information Technology, pp. 498-503, 2008.

[18]N. Brahmi, M. Boussedjra and J. Mouzna,. "Routing in Vehicular Ad Hoc Networks: towards RoadConnectivity Based Routing", Mobile Ad-Hoc Networks: Applications, Xin Wang (Ed.), ISBN: 978953-307-416-0, 2011.

[19] Q. Chen, F. Schmidt-Eisenlohr, D. Jiang,. "Overhaul of ieee 802.11 modeling and simulation in ns2". InProceedings of the 10th ACM Symposium on Modeling, analysis, and simulation of wireless and mobile systems (MSWiM '07). ACM, New York, NY, USA, 159-168, 2007.

[20]T. Clausen, and P. Jacquet, "Optimized Link State Routing Protocol (OLSR)". IETF RFC 3626, [online] Available in URL http://www.ietf.org/rfc/rfc3626.txt, 2003.

[21]W. C. William, "VoIP Service Quality Measuring and Evaluating Packet-Switched Voice". 1st edition. USA: McGraw-Hill Networking Professional, 2002.

[22]T. Murray, M. Cojocari, and H. Fu, "Measuring the performance of IEEE 802.11p using NS-2 simulator for vehicular networks," in IEEE International Conference on Electro/Information Technology, pp. 498-503, 2008.

[23] D.V. Viswacheda, L. Barukang, M.Y Hamid,. and M.S. Arifianto, "Performance Evaluation of Mobile Ad Hoc Network Based Communications for Future Mobile Tele-Emergency System”, Journal of Applied Sciences Volume 7, Number 15, 2111-2119, 2007. 